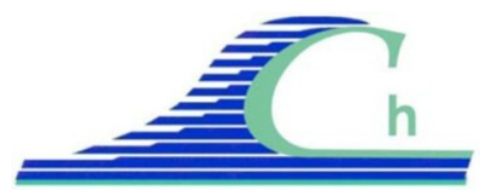

XII ${ }^{\text {ìmes }}$ Journées Nationales Génie Côtier - Génie Civil

Cherbourg, 12-14 juin 2012

DOI:10.5150/jngcgc.2012.082-N @ Editions Paralia CFL

disponible en ligne - http://www.paralia.fr - available online

\title{
Solution de renforcement des ouvrages maritimes vis-à-vis de la remontée du niveau des mers
}

\author{
Dang Trinh NGUYEN ${ }^{1}$, Jérôme BROSSARD ${ }^{1}$
}

1. Laboratoire Ondes et Milieux Complexes, UMR 6294, CNRS-Université du Havre,

53 rue de Prony, BP 540, 76058 Le Havre Cedex, France.

bachkhoaman85@yahoo.com ; jerome.brossard@univ-lehavre.fr

\section{Résumé :}

La surélévation du niveau des mers associée aux changements climatiques nécessite d'envisager des solutions de renforcement des ouvrages de protection maritimes. Diverses solutions sont testées en canal à houle avec comme objectifs de conserver les débits de franchissement de projet pour un scénario "moyen" et un scénario "extrême" de surélévation du niveau moyen des mers et océans de $0,5 \mathrm{~m}$ et de $1 \mathrm{~m}$.

Mots-clés : Digues maritimes - Franchissement ouvrages - Renforcements ouvrages

\section{Introduction}

Une des conséquences attendues du changement climatique est la remontée du niveau des mers et océans. Le 4ème rapport d'évaluation du GIEC (2007) synthétisé par l'ONERC (2010) propose un scénario dit "optimiste" de surélévation de 0,40 $\mathrm{m}$ et un scénario "pessimiste" avec une surélévation de 0,60 m à l'horizon 2100. Certains scénarios dits "extrêmes" proposent des surélévations possibles de 1 à $2 \mathrm{~m}$. Cette variation de niveau moyen, en elle-même, et les effets induits sur l'augmentation des hauteurs de vagues à l'ouvrage conduisent à des franchissements accrus des ouvrages maritimes de protection et donc à des aléas d'inondation ainsi qu'à des aléas sur l'exploitation des zones abritées non pris en compte dans les cahiers des charges de conception initiaux. Dès lors la question du renforcement des ouvrages existants se pose. Celle ci a fait l'objet, en 2010 et 2011 du programme national GICC-SAOPOLO (GICC : Gestion et Impacts du Changement Climatique, programme piloté par le Ministère de 1'Ecologie, du Développement Durable, des Transports et du Logement; SAOPOLO : Stratégies d'adaptation des ouvrages de protection marine ou des modes d'occupation du littoral vis-à-vis de la montée du niveau des mers et des océans, coordonné par le CETMEF).

Un recensement des ouvrages de protection réalisé dans le cadre de ce programme a mis en évidence que plus des trois quarts des ouvrages sont des digues à talus (Chapitre $1 \mathrm{du}$ rapport provisoire SAOPOLO en cours de finalisation). Parmi ceux-ci le programme a distingué les ouvrages dits "maritimes" et les ouvrages côtiers comme les hauts de plage. Les moyens et la durée du programme étant limités il a été décidé d'étudier les perrés imperméables et les carapaces en enrochement dans le cadre des ouvrages côtiers 
et les carapaces en blocs artificiels dans le cas des digues maritimes implantées dans des zones à plus grande profondeur. Pour ces différents ouvrages, des bathymétries et des sections types ont été définies. Celles-ci ont été jugées représentatives des ouvrages du littoral français par des experts participant au programme (Chapitre $1 \mathrm{du}$ rapport provisoire SAOPOLO).

Dans le cadre de ce programme le Laboratoire Ondes et Milieux Complexes de l'université du Havre a été chargé d'étudier expérimentalement des solutions de renforcement d'un ouvrage maritime type pour un scénario de surélévation dit "moyen" de $0,5 \mathrm{~m}$ et un scénario "extrême" de $1 \mathrm{~m}$.

\section{Ouvrage de référence et conditions expérimentales}

L'objectif d'étudier différentes solutions de renforcement de digues maritimes existantes nous a conduit à choisir comme ouvrage de référence type une digue à talus avec carapace en blocs artificiels type BCR (blocs cubiques rainurés dits aussi d'Antifer). Ce type d'ouvrage nous a semblé assez représentatif du point de vue de la variété des digues existantes et des solutions de renforcement envisagées. De même, une profondeur d'eau en pied d'ouvrage de $\mathrm{h}=7 \mathrm{~m}$ pour le niveau moyen de référence a été jugée représentative de l'ensemble des digues maritimes françaises.

Plutôt que de partir des plans d'un ouvrage existant, nous avons choisi de dimensionner une digue "virtuelle", dite de référence, en utilisant les règles de l'art actuelles à partir de conditions de bathymétrie et de climat de houle donnés.

Les dimensions des matériaux utilisés autant que la cote d'arase de la digue sont déterminés par les points de vue franchissement et stabilité de la carapace.

La figure 1 présente la section de référence de la digue.

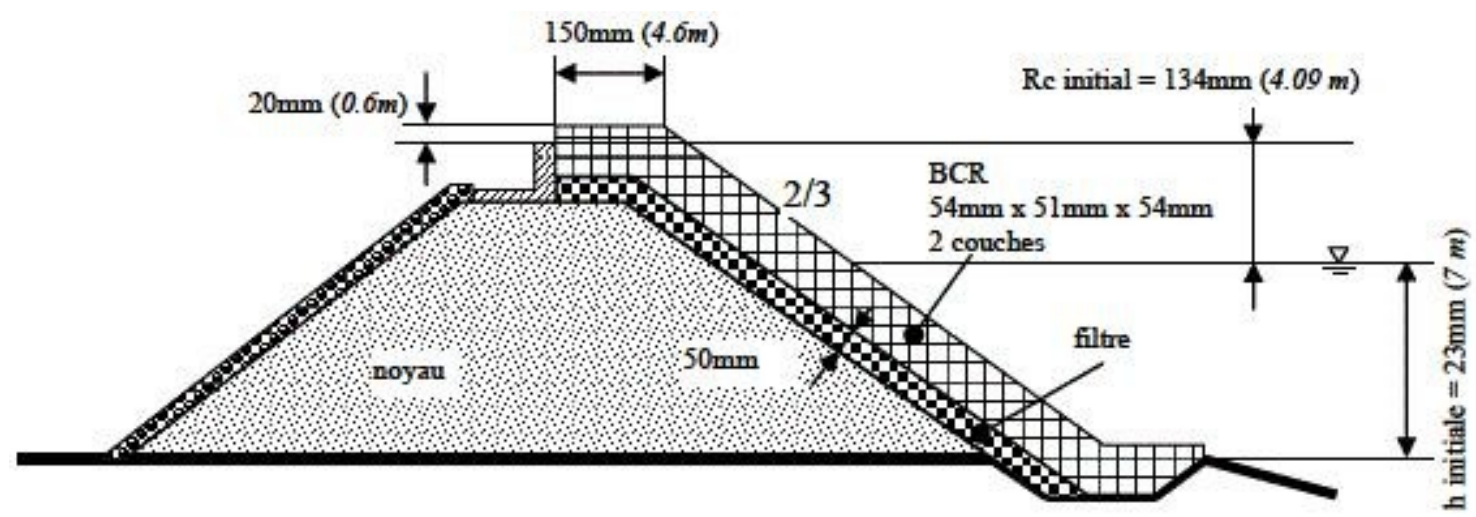

Figure 1. Section droite de la digue (en italique : valeurs à l'échelle nature).

Afin de comparer les résultats expérimentaux obtenus avec les modèles semiempiriques (franchissement avec OWEN (1980) et stabilité de la carapace avec VAN DER MEER (1988)) utilisés aujourd'hui par les concepteurs d'ouvrages et, 


\section{XII ${ }^{\text {èmes }}$ Journées Nationales Génie Côtier - Génie Civil \\ Cherbourg, 12-14 juin 2012}

éventuellement, de proposer des modifications de ces modèles prenant en compte les renforcements, nous avons choisi de ne pas seulement réaliser des mesures correspondant aux conditions extrêmes de houle mais de faire des campagnes de mesures pour quatre valeurs de périodes moyennes $T_{m}$ et plusieurs hauteurs $H_{s}$ de houle pour chacune de ces périodes. Les plages de variation de ces grandeurs ont été supposées représentatives par l'ensemble des participants au programme SAOPOLO des climats de houle auxquels sont soumis les ouvrages maritimes.

Les conditions expérimentales sont :

- période moyenne : $7 \mathrm{~s} \leq \mathrm{T}_{\mathrm{m}} \leq 11 \mathrm{~s}$;

- hauteur significative (houle au large) : $5 \mathrm{~m} \leq \mathrm{H}_{\mathrm{s} 0} \leq 10 \mathrm{~m}$;

- pente des fonds devant l'ouvrage : $2 \%$;

- hauteur d'eau initiale, de référence, en pied d'ouvrage : $h=7 \mathrm{~m}$;

- durée des essais : $3 \mathrm{~h}$.

Les essais de franchissement sont réalisés dans un canal à houle de $26 \mathrm{~m}$ de long, 0,88 $\mathrm{m}$ de large et 1,2 $\mathrm{m}$ de hauteur d'où une échelle géométrique pour les essais de 1/30,5.

Les volumes de franchissement sont récupérés dans un bac en arrière de l'ouvrage s'étendant sur toute la largeur du canal. Une pompe permet de vider en continu le bac avec réinjection en amont afin de conserver le niveau d'eau dans le canal. Une mesure des volumes de franchissement est assurée par des compteurs à eau. Le débit moyen de franchissement par mètre linéaire $\left(\mathrm{m}^{3} / \mathrm{m} / \mathrm{s}\right)$ est déterminé par :

$q=\frac{V}{t B}$

où $V$ est le volume total d'eau ayant franchi la digue durant la durée de l'essai, $t$ est la durée de l'essai et $B$ la largeur du canal.

\section{Effets d'une surélévation du niveau moyen de $0,5 \mathrm{~m}$ sur les franchissements}

Des essais ont été réalisés pour mesurer les effets d'une élévation du niveau moyen de $0,5 \mathrm{~m}$ sur les franchissements pour la digue de référence sans renforcement. La hauteur d'eau passe donc de $7 \mathrm{~m}$ à 7,5 $\mathrm{m}$ en pied d'ouvrage. La figure 2 présente les résultats obtenus pour les quatre périodes testées.

Les débits de franchissement sont fortement accrus pour cette surélévation de $0,5 \mathrm{~m}$ du niveau moyen. Les formules semi-empiriques de franchissement (par exemple : OWEN, 1980 ; BESLEY, 1999 ; VAN DER MEER, 1998) montrent qu'effectivement le débit de franchissement croît exponentiellement avec la diminution du franc bord $R_{c}$ de l'ouvrage.

L'objectif de l'étude était d'imaginer des solutions de renforcement permettant de retrouver des volumes de franchissement de référence pour ce cas de surélévation "moyen".

\section{Renforcement pour un scénario "moyen": $\Delta h=0,5 \mathrm{~m}$}


Les tests réalisés avec différentes valeurs de rehausse du mur de couronnement, nous conduisent, pour limiter les débits de franchissement aux valeurs de référence, à proposer un accroissement de hauteur du mur de $1,5 \mathrm{~m}$ pour cette surélévation du niveau moyen de $0,5 \mathrm{~m}$.

La figure 2 donne les évolutions des débits de franchissement obtenus avec le renforcement proposé en comparaison avec l'état de référence $(\mathrm{h}=7 \mathrm{~m})$ et le comportement de la digue non renforcée avec une surélévation du niveau moyen de $0,5 \mathrm{~m}$.

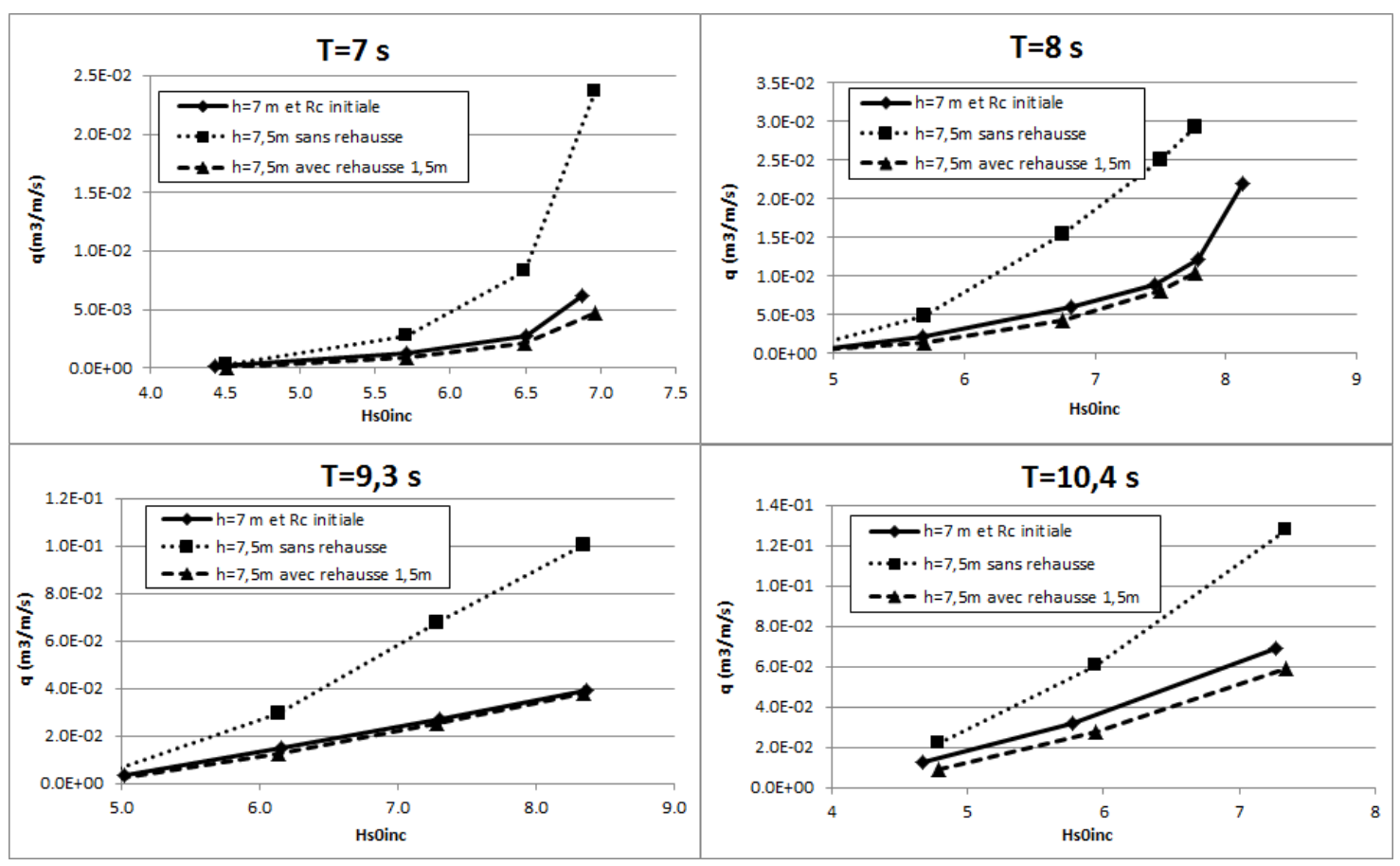

Figure 2. Comparaison des débits de franchissement pour : l'état de référence et une surélévation de 0,5 m sans et avec une rehausse du mur de couronnement de 1,5 m.

Il faut noter que les résultats présentés ici sont relatifs aux couples $\left(\mathrm{H}_{\mathrm{s}}, \mathrm{T}_{\mathrm{m}}\right)$ incidents déduits des spectres incidents et réfléchis mesurés juste devant l'ouvrage. Des consignes identiques imposées au générateur de houle pour les différents tests ne conduisent pas exactement aux mêmes couples $\left(\mathrm{H}_{\mathrm{s}}, \mathrm{T}_{\mathrm{m}}\right)$ incidents à cause de conditions de réflexion de l'ouvrage légèrement différentes suivant le niveau d'eau et les multi-réflexions entre batteur et ouvrage.

Avec ce renforcement par rehaussement du mur de couronnement de 1,5 m les débits de franchissement sont voisins de ceux obtenus dans les conditions de référence sur les pages de variation de hauteurs et de périodes de vagues.

Dans ces conditions, nous avons constaté que la stabilité de la carapace était assurée.

\section{Renforcement pour un scénario "extrême" : $\Delta h=1 \mathrm{~m}$}




\section{XII ${ }^{\text {èmes }}$ Journées Nationales Génie Côtier - Génie Civil \\ Cherbourg, 12-14 juin 2012}

Contrairement au cas précédent, il a été impossible, pour la plupart des tests, de mesurer les débits de franchissement avec la digue non renforcée et la surélévation de $1 \mathrm{~m}$. En effet, ceux-ci étaient beaucoup trop importants pour être "évacués" suffisamment vite par le système réservoir de récupération-pompe; des volumes de plus de $2 \mathrm{~m}^{3}$ peuvent être atteints sur la durée d'un essai de l'ordre de $2000 \mathrm{~s}$ (échelle modèle).

Pour ce scénario six solutions de renforcement ont été envisagées.

\subsection{Rehausse du mur de couronnement avec ou sans béquet de déflexion}

Pour cette condition "extrême" nous avons constaté qu'un rehaussement du mur de couronnement de $2,1 \mathrm{~m}$ avec un béquet de déflexion permettait de limiter les franchissements aux valeurs de référence. Cependant, ce type de solution accroît fortement la réflexion sur l'ouvrage et engendre des vitesses importantes autour des blocs de la carapace. Ces phénomènes induisent alors une augmentation importante des mouvements des blocs conduisant à des taux d'endommagement dépassant les seuils admissibles.

Ce constat conduit à envisager d'autres solutions de renforcement qui suivent deux logiques différentes : diminution de l'énergie de la houle à l'ouvrage en construisant une digue détachée submergée en amont de l'ouvrage ou renforcement de la carapace de la digue principale par ajout de blocs soit sous forme de berme en pied d'ouvrage soit sous forme d'une troisième couche.

\subsection{Digue détachée avec immersion de $3,7 \mathrm{~m}$}

Cette configuration, présentée sur la figure 3 , ne permet pas de réduire suffisamment les franchissements. Une variante consiste à augmenter la hauteur de la digue détachée.

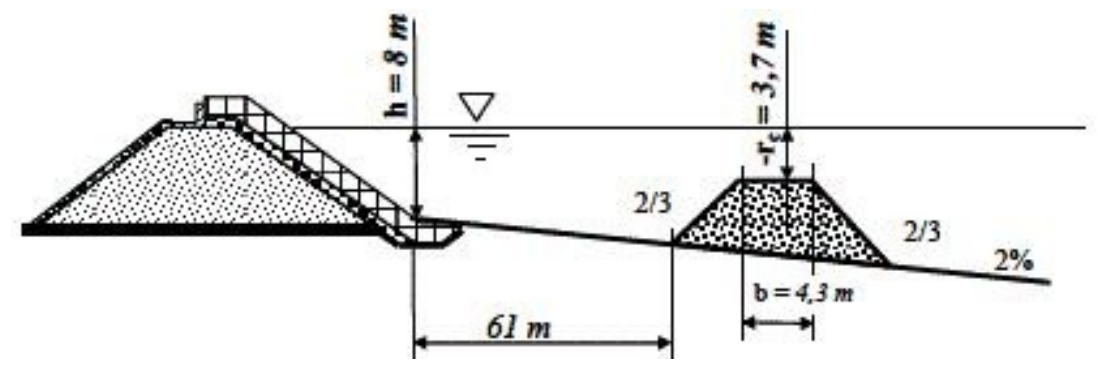

Figure 3. Configuration avec digue détachée immergée à 3,7 m.

\subsection{Digue détachée avec immersion de $1,98 \mathrm{~m}$}

La réduction d'immersion accroît les risques d'instabilité par érosion du matériau constitutif; d'où le choix d'utiliser le même matériau que précédemment comme noyau mais recouvert d'une couche de blocs artificiels (type ANTIFER) identiques à ceux de la carapace de la digue principale. Cette configuration permet de réduire l'immersion à $1,98 \mathrm{~m}$. 
Cette configuration ne permet pas non plus d'atteindre les valeurs initiales de franchissement.

\subsection{Berme en pied d'ouvrage}

Afin de rendre économiquement comparable cette solution avec la précédente nous avons choisi d'utiliser les mêmes blocs artificiels et en même nombre que précédemment (figure 4).

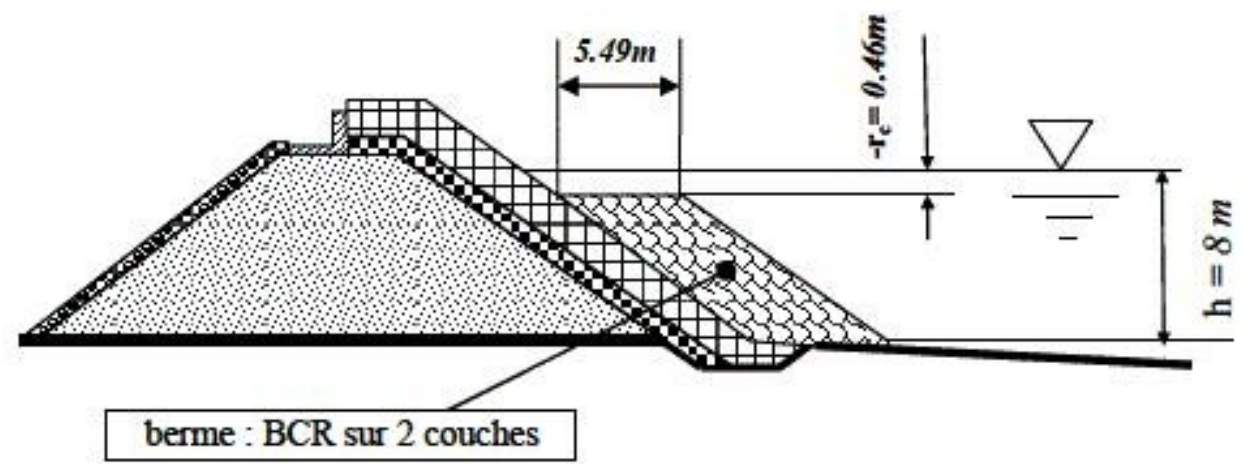

Figure 4. Profil de la digue principale avec une berme de BCR sur 2 couches.

Cette configuration ne permet pas non plus de réduire suffisamment le franchissement.

\subsection{Troisième couche de blocs artificiels sur la carapace}

Toujours pour mieux comparer les différentes solutions nous avons choisi de placer les mêmes blocs artificiels et en même nombre que précédemment pour constituer une troisième couche pour la carapace.

Cette solution permet de réduire les franchissements de façon plus efficace que les solutions précédentes (digue détachée et berme); cependant les débits restent encore supérieurs aux valeurs de référence.

Une variante consiste à rendre le haut de carapace moins perméable en surélevant le mur de couronnement.

5.6 Troisième couche de blocs artificiels sur la carapace et rehausse du mur de couronnement

Une rehausse jusqu'au niveau supérieur de la carapace permet d'atteindre les objectifs visés concernant le franchissement et la stabilité de la carapace (figure 5).

La figure 6 montre que sur toute la plage de périodes testées, les volumes de franchissement obtenus avec cette solution de renforcement sont inférieurs aux valeurs correspondant à la configuration de référence. 


\section{XII ${ }^{\text {èmes }}$ Journées Nationales Génie Côtier - Génie Civil \\ Cherbourg, 12-14 juin 2012}

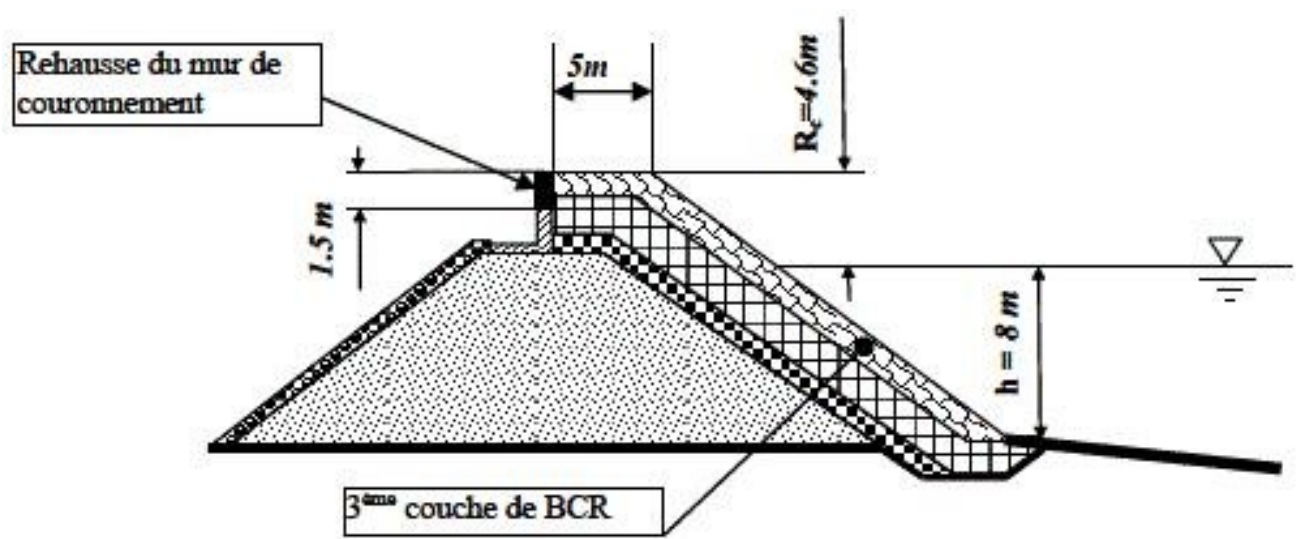

Figure 5. Digue avec carapace de 3 couches de BCR et rehausse du mur de couronnement de 1,5 m.

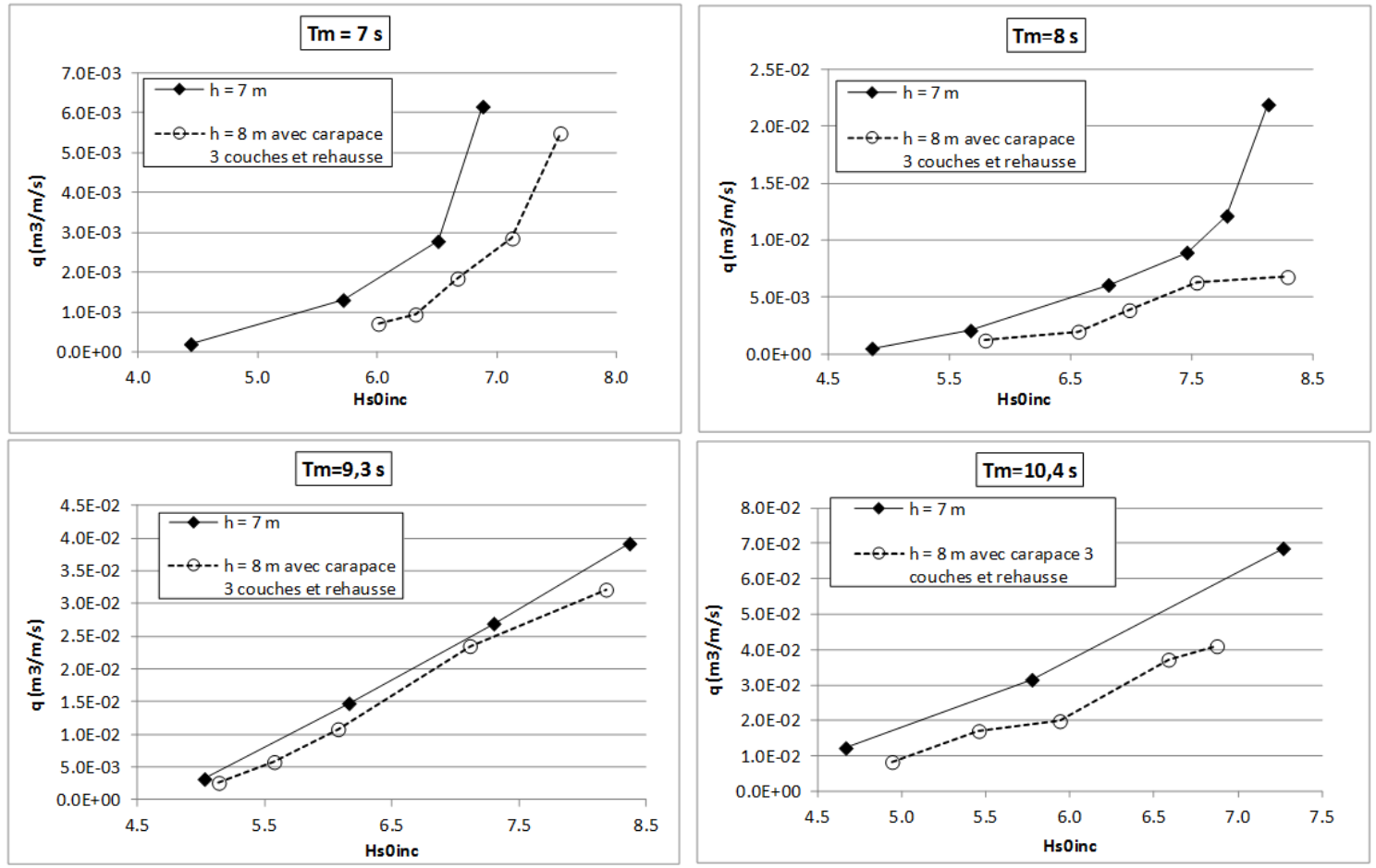

Figure 6. Débits de franchissement pour une carapace à 3 couches de BCR et une rehausse du mur de couronnement de $1,5 \mathrm{~m}$

$\mathrm{Au}$ vu des résultats on pourrait envisager des blocs moins. Cependant la marge doit être relativement faible pour deux raisons. D'une part des blocs de dimension moindre pour la troisième couche réduirait la porosité et donc la dissipation d'énergie et donc favoriserait les franchissements. D'autre part des blocs de poids moindre poseraient probablement des problèmes de stabilité.

\section{Conclusion}

De la modélisation physique réalisée on peut déduire qu'une solution de renforcement vis-à-vis du franchissement par une rehausse du mur de couronnement convient pour 
des surélévations du niveau moyen ne dépassant pas $0,5 \mathrm{~m}$ ce qui entre dans le cadre des prévisions actuelles à l'échelle du siècle. L'intérêt de cette solution réside dans son application pouvant être progressive dans le temps. Deux problèmes restent à résoudre : le dimensionnement de la modification du mur de couronnement du point de vue de sa résistance structurelle et l'acceptation d'une plus grande obstruction de l'horizon. Pour des surélévations de niveau moyen "extrêmes", des solutions ayant des conséquences structurelles sur l'ensemble de l'ouvrage doivent être envisagées. Le renforcement de la seule partie supérieure de l'ouvrage ne semble pas suffisant pour conserver l'intégrité de l'ouvrage; l'augmentation de l'épaisseur de la carapace par une troisième couche d'enrochement constitue une solution viable.

L'objectif principal du programme SAOPOLO est de fournir aux maîtres d'ouvrages des indications sur des solutions de renforcement des ouvrages vis-à-vis de la remontée du niveau moyen des mers. Ainsi, une partie du programme est consacrée à l'évaluation des coûts économiques et sociétaux associés aux inondations, donc aux franchissements. Une telle analyse des coûts de renforcement par rapport aux bénéfices doit s'appuyer sur des modèles prédictifs de franchissement tels ceux d'OWEN (1980) ou de VAN DER MEER (1998) par exemple. A partir des essais physiques réalisés, en partie rapportés ici, nous avons pu mettre en évidence une relative inadaptation de ces modèles semi-empiriques pour les cas de forts franchissements. Des propositions de modifications de ces modèles, non présentées ici, ont été élaborées pour des configurations de digues en enrochement à faible cote d'arase.

\section{Références bibliographiques}

BESLEY P. (1999). Wave overtopping of Seawalls. Design and Assessment Manual. Hydraulics research Wallingford. R\&D Technical Report W178.

GIEC (2007). Bilan 2007 des changements climatiques. Contribution des Groupes de travail I, II et III au quatrième Rapport d'évaluation du GIEC sur l'évolution du climat [Équipe de rédaction principale, Pachauri, R.K. et Reisinger, A.]. GIEC, Genève, Suisse, 103 p.

ONERC (2010). Prise en compte de l'élévation du niveau de la mer en vue de l'estimation des impacts du changement climatique et des mesures d'adaptation possibles. Synthèse $\mathrm{n}^{\circ} 2$, février 2010, disponible sur :

URL : http://www.developpement-durable.gouv.fr/IMG/pdf/synth_niveau_mer.pdf OWEN M.W. (1980). Design of seawalls allowing for wave overtopping. Hydraulics Research, Wallingford, Report No. EX 924, UK.

VAN DER MEER J. W. (1988). Stability of cubes, tetrapods and Accropode. Design of breakwaters. Proc. Conf. breakwaters'88, Eastbourne, 4-6 mai 1988, Londes, pp 59-68. VAN DER MEER J. W. (1998). Wave Run-Up and Overtopping. Chapter 8 in: "Dikes and Revetments: Design, Maintenance and Assessment". Published by A.A. Balkema. 